\section{Humanized zebrafish enhance human hematopoietic stem cell survival and promote acute myeloid leukemia clonal diversity}

\author{
Vinothkumar Rajan, ${ }^{1}$ Nicole Melong, ${ }^{2}$ Wing Hing Wong, ${ }^{3}$ Benjamin King, ${ }^{4}$ R. \\ Spencer Tong, ${ }^{5}$ Nitin Mahajan, ${ }^{3}$ Daniel Gaston, ${ }^{6}$ Troy Lund, ${ }^{7}$ David Rittenberg, ${ }^{8}$ \\ Graham Dellaire, ${ }^{9}$ Clinton J.V. Campbell, ${ }^{10}$ Todd Druley, ${ }^{3}$ and Jason N. Berman ${ }^{1,2,11^{*}}$ \\ ${ }^{1}$ Department of Microbiology and Immunology, Dalhousie University, Halifax, Nova \\ Scotia, Canada; ${ }^{2}$ Department of Pediatrics, University of Ottawa, Ottawa, Ontario, \\ Canada; ${ }^{3}$ Department of Pediatrics, Division of Hematology-Oncology, Washington \\ University, St. Louis, MO, USA; ${ }^{4}$ Department of Ocean Sciences, Memorial University, St. \\ John's, Newfoundland and Labrador, Canada; ${ }^{5} \mathrm{MRC}$ Weatherall Institute of Molecular \\ Medicine, University of Oxford, John Radcliffe Hospital, Oxford, UK; ${ }^{6}$ Department of \\ Pathology, Dalhousie University, Halifax, Nova Scotia, Canada; ${ }^{7}$ Department of \\ Pediatrics, University of Minnesota, Minneapolis, MN, USA; ${ }^{8}$ Department of Obstetrics \\ and Gynecology, IWK Health Science Center, Halifax, Nova Scotia, Canada; \\ ${ }^{9}$ Departments of Pathology and Biochemistry \& Molecular Biology, Dalhousie University, \\ Halifax, Nova Scotia, Canada; ${ }^{10}$ Stem Cell and Cancer Research Institute, McMaster \\ University, Hamilton, Ontario, Canada and ${ }^{11} \mathrm{CHEO}$ Research Institute, Ottawa, Ontario, \\ Canada
}

\section{SFerrata Storti Foundation}

Haematologica 2020

Volume 105(10):2391-2399

\title{
ABSTRACT
}

X enograft models are invaluable tools in establishing the current paradigms of hematopoiesis and leukemogenesis. The zebrafish has emerged as a robust alternative xenograft model but, like mice, lacks specific cytokines that mimic the microenvironment found in human patients. To address this critical gap, we generated the first "humanized" zebrafish that expresses human hematopoietic-specific cytokines (granulocyte-monocyte colony-stimulating factor, stem cell factor, and stromal cell-derived factor $1 \alpha$ ). Termed GSS fish, these zebrafish promote survival, self-renewal and multilineage differentiation of human hematopoietic stem and progenitor cells and result in enhanced proliferation and hematopoietic niche-specific homing of primary human leukemia cells. Using error-corrected RNA sequencing, we determined that patient-derived leukemias transplanted into GSS zebrafish exhibit broader clonal representation compared to transplants into control hosts. GSS zebrafish incorporating error-corrected RNA sequencing establish a new standard for zebrafish xenotransplantation which more accurately recapitulates the human context, providing a more representative cost-effective preclinical model system for evaluating personalized response-based treatment in leukemia and therapies to expand human hematopoietic stem and progenitor cells in the transplant setting.

\section{Introduction}

The availability of xenograft models has dramatically influenced our current understanding of leukemogenesis and stem cell biology over the last decade. Patientderived xenografts provide a better microenvironmental and stromal context than any in vitro system because they maintain the clonal heterogeneity inherent in human cancers, which is of translational importance for assays that involve pharmacological interventions and responses. ${ }^{1,2}$ Current gold standard xenograft assays use small mammals, like the mouse, with a depleted immune system in models that have been refined over many years from their original derivation. ${ }^{3 \cdot 6}$ However, findings from these murine xenografts may not be congruent with similar experimental results observed in human studies.? Some human samples do not engraft in a foreign host,

\section{Correspondence:}

JASON N. BERMAN

jberman@cheo.on.ca

Received: March 27, 2019.

Accepted: December 5, 2019.

Pre-published: December 19, 2019.

doi:10.3324/haematol.2019.223040

\section{(C)2020 Ferrata Storti Foundation}

Material published in Haematologica is covered by copyright. All rights are reserved to the Ferrata Storti Foundation. Use of published material is allowed under the following terms and conditions:

https://creativecommons.org/licenses/by-nc/4.0/legalcode. Copies of published material are allowed for personal or internal use. Sharing published material for non-commercial purposes is subject to the following conditions: https://creativecommons.org/licenses/by-nc/4.0/legalcode, sect. 3. Reproducing and sharing published material for commercial purposes is not allowed without permission in writing from the publisher. 
while in other cases, following successful initial engraftment, the chimera disappears over time. Given that xenografts include both human tumor and host stroma (including immune cells), these discrepancies are accounted for, in part, by the lack of evolutionary conservation of microenvironmental signaling pathways between humans and rodents.

Further, cytokines present in the microenvironment are essential for the differentiation and maintenance of individual cells but are not entirely conserved across species. ${ }^{8}$ For example, there is a lack of conservation of interleukin 3 (IL3 ) and granulocyte-macrophage colony stimulating factor (GM-CSF/CSF2) between humans and mice at the amino acid level, evidenced by the fact that mouse IL-3 and GMCSF do not react with their respective human receptors. ${ }^{9,10}$ Thus, to compensate for these limitations, efforts to "humanize" rodent model systems have led to the introduction of human microenvironmental factors along with human cell populations. ${ }^{11,12}$

Various efforts have been made to introduce human factors into model organisms, including the injection of recombinant proteins such as PIXY321 (a GM-CSF/IL-3 fusion protein) ${ }^{4}$ and a cost-efficient method to enable human cytokine expression using knock-in ${ }^{10,13}$ and transgenic technologies, ${ }^{11,14}$ where researchers have introduced various factors including erythropoietin and IL-3. The approach of humanizing mice has been successful to the extent that it permits enhanced engraftment and, depending on the cytokine introduced, differentiation and maintenance of specific cell lineages. For example, humanized transgenic SGM3 mice expressing human stem cell factor/KIT ligand (SCF/KITLG), GM-CSF and IL-3 showed a significant increase in the myeloid ${ }^{15}$ and mast cell compartments ${ }^{16}$ and improved engraftment efficiency of human acute myeloid leukemia (AML) cells. ${ }^{11}$ This modified murine xenograft model provides a unique advantage to enhance clonal heterogeneity and thereby enrich for more robust and meaningful responses to pharmacological interventions. However, the mouse model has significant limitations: it remains laborious, is limited to small numbers of animals, and human cells take months to engraft. As such, they are not amenable to high- or medium-throughput drug screening efforts and cannot provide results to inform patient management decisions in a clinically actionable time-frame.

We previously pioneered a zebrafish larval xenograft assay to study human leukemia progression and demonstrated the feasibility of employing this platform for primary patient bone marrow-derived T-cell acute lymphoblastic leukemia (T-ALL) samples. ${ }^{17-19}$ The zebrafish xenograft platform offers several advantages, including a high degree of genetic conservation with humans at the protein level ${ }^{20}$ with the added benefit of visual tractability of human cells in an organism amenable to medium-throughput chemical screening. ${ }^{21,22}$ However, similar to mice, zebrafish express evolutionarily divergent cytokines (or lack them altogether) that are critical to the maintenance of human cell clonal heterogeneity. Previous publications have suggested that the receptors and ligands of the IL-3 subfamily that include IL-5, GM-CSF, and IL-3 are absent in zebrafish, ${ }^{23}$ and in silico analysis revealed that the critical cell migration chemokine, CXCL12/SDF1 $\alpha$, is conserved less than $50 \%$ at the amino acid level between humans and zebrafish.

While zebrafish leukemia xenograft platforms have been successful, ${ }^{17,18}$ the survival of human hematopoietic stem and progenitor cells (HSPC) in zebrafish is uncertain. It was previously demonstrated that HSPC do not survive in zebrafish for more than 12 hours, ${ }^{24}$ while a recent study showed that they could survive only up to 13 hours postinjection, ${ }^{25}$ raising concerns whether the zebrafish host enable human HSPC survival and clonal expansion after transplantation. As such, zebrafish xenograft approaches to date share a flaw in lacking an optimal microenvironment to support the clonal evolution of human HSPC and leukemia cells, questioning the clinical transferability of findings from this model. To address this critical gap, we have generated a humanized zebrafish that expresses multiple human hematopoietic-specific cytokines. We subsequently transplanted primary human-derived HSPC and leukemia cells and performed clonal heterogeneity evaluation using error-corrected sequencing (ECS). Using these humanized zebrafish models, we reveal that transgenic fish expressing human cytokines prolong survival and differentiation of human HSPC. Furthermore, in the presence of these critical cytokines, transplanted leukemia cells exhibit hematopoietic niche homing that more accurately models the behavior of human leukemia. These results lay the foundation for a new paradigm in zebrafish xenograftbased drug discovery platforms for molecular targeting of human leukemia and the expansion of HSPC.

\section{Methods}

\section{Zebrafish studies}

All zebrafish studies reported were approved by the Dalhousie University Committee on Laboratory Animals (UCLA), under protocol \#17-007. Briefly, the tol2 constructs were injected into singlecell stage zebrafish to make the human CXCL12/SDF1 $\alpha$ and the human SCF/KITLG and GM-CSF/CSF2-expressing transgenic zebrafish. Fish were further crossed to make a humanized triple GSS (GM-CSF, SCF, and SDF1 $\alpha$ ) transgenic fish (see details in the Online Supplementary Methods). The casper ${ }^{26}$ strain of double pigment zebrafish mutants was used to generate all transgenic and control fish.

\section{Human umbilical cord and bone marrow samples}

The use of human samples in the study was approved by the IWK Health Center Research Ethics Board (REB\# 1007549 \& REB\# 1007549). Fresh human umbilical cord blood and human leukemia bone marrow samples were collected from patients at IWK Health Center (Halifax, Nova Scotia, Canada) after formal consent had been obtained. For leukemia samples, the buffy coat was isolated, and the white blood cells were cryopreserved directly. For samples from umbilical cord blood, post-buffy coat isolation, the samples underwent immunomagnetic enrichment to isolate lineage-depleted (lin)HSPC.

\section{Orthotropic xenograft experiments with primary samples}

Both transgenic and control (casper) larvae were treated with 10 $\mu \mathrm{g} / \mathrm{mL}$ doxycycline hydrochloride (Sigma Aldrich) at 24 hours post-fertilization (hpf) to induce the expression of SCF/KITLG and GM-CSF/CSF2 in transgenic fish and as a control for any drug effects in casper larvae. All larvae were irradiated at $72 \mathrm{hpf}$ to induce $\mathrm{cxcl} 12$ promoter activity and niche clearance of the organism for transplant. Human patient-derived samples were then labeled with a cytoplasmic green fluorescent dye to facilitate in vivo cell tracking, and approximately 150-250 cells were injected into the common cardinal vein. The larvae were screened immediately 
following the injection to confirm that cells were present in the circulation and then moved to a $35^{\circ} \mathrm{C}$ incubator, a previously-established compromise between the normal developing temperature of zebrafish $\left(28.5^{\circ} \mathrm{C}\right)$ and human cells $\left(37^{\circ} \mathrm{C}\right) .{ }^{18,27}$ Detailed methods are provided in the Online Supplementary Methods.

\section{Results}

\section{Generating "humanized" transgenic zebrafish}

To improve the current zebrafish platform for human leukemia and HSPC xenografts, we generated transgenic zebrafish expressing human hematopoietic cytokines. Cytokines that are poorly conserved between human and zebrafish but that have been demonstrated to be critical for normal hematopoiesis were chosen for this study. In this regard, the CXCR4 ligand, CXCL12/SDF1 $\alpha$, was our priority, given its functions in stem cell fate decisions such as expansion, homing, self-renewal, differentiation, control of stem cell exhaustion and protection against genotoxic stress. ${ }^{28-30}$ Both GM-CSF/CSF2 and SCF/KITLG were also determined to be essential candidates based on previous mouse experiments. ${ }^{11}$ Due to its redundant function with

A

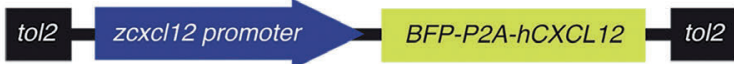

B

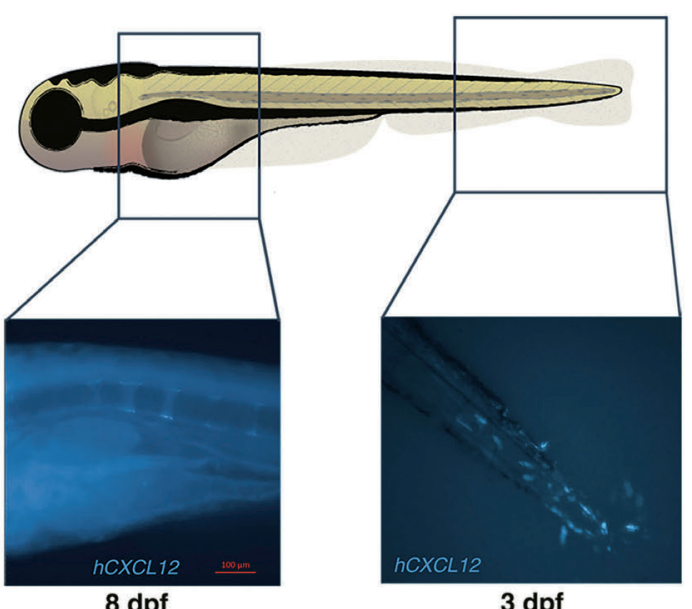

C
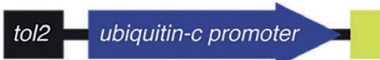
rTA
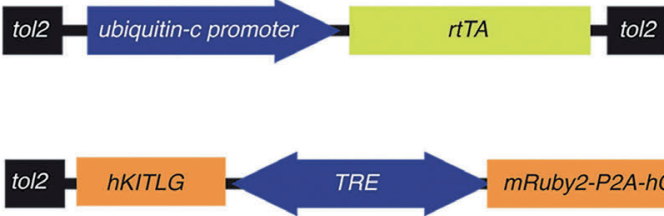

CXCL12 and GM-CSF/CSF2, we did not incorporate IL-3, which was previously used in mouse models. We developed two independent transgenic zebrafish models by coinjecting tol2 mRNA and plasmids (Figure 1A, C). One expresses human CXCL12 under the zebrafish cxcl12 promoter (Figure 1B) while the other expresses human KITLG and CSF2 under a tetracycline-inducible promoter (Figure 1D); expression was confirmed using immunoblotting (Figure 1E).

\section{Humanized zebrafish demonstrate enhanced human Ieukemia cell migration and proliferation}

We initially performed validation experiments in the CXCL12 and GM-CSF/CSF2-SCF/KITLG compound transgenic models separately. The CXCR4-CXCL12 axis is critical for cell migration and homing. ${ }^{31}$ We therefore selected migration as a mode of validation for the human CXCL12expressing transgenic zebrafish. Jurkat cells are a human TALL cell line that expresses high levels of CXCR4, the cognate CXCL12 receptor. ${ }^{32}$ Zebrafish $\mathrm{cxCl12}$ promoter expression begins only at $72 \mathrm{hpf}$, so we injected Jurkat cells into the yolk sac of CXCL12-expressing and casper control larvae at $72 \mathrm{hpf}$ and screened for migration at 3 days post-injection
D

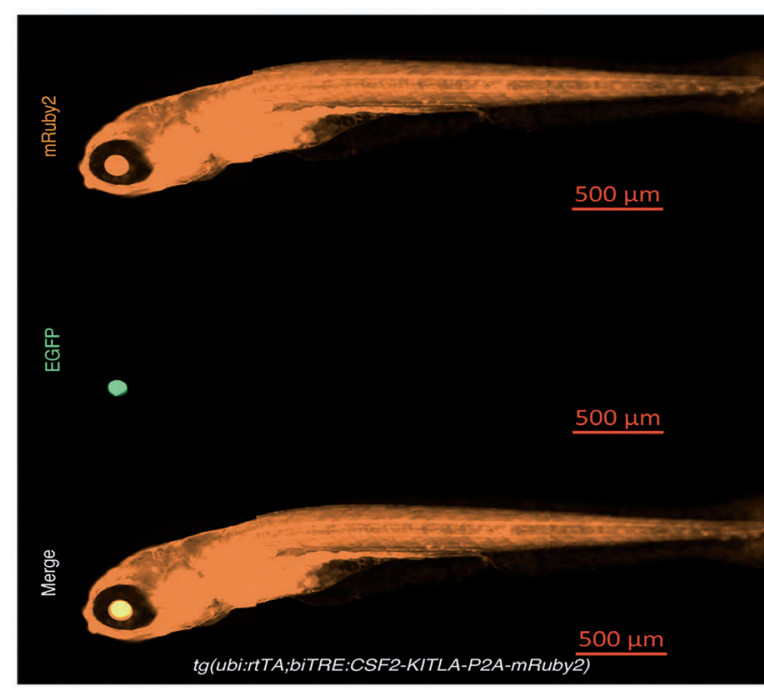

E

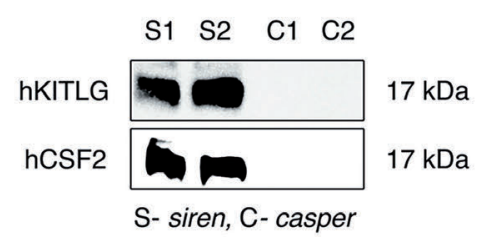

Figure 1. Humanized transgenic zebrafish express human CXCL12, KITLG, and CSF2. Transgenic zebrafish expressing human cytokines were generated by co-injecting tol2 mRNA and plasmids (A and C). (A) A cartoon of the construct used to make the transgenic zebrafish expressing human CXCL12 (hCXCL12) along with tagBFP under the zebrafish cxcl12 promoter. (B) Representative image of a transgenic zebrafish expressing human CXCL12 in the posterior hemal arc near the tip of the tail at 3 days post-fertilization (dpf). CXCL12 expression continues to progress anteriorly through the hemal arc (representative image shows 8 dpf larvae). (C) A cartoon of the constructs used to make the tet-inducible human SCF/KITLG and GM-CSF/CSF2 expressing zebrafish. (D) Representative image of the human SCF/KITLG (hKITLG) and GM-CSF/CSF2 (hCSF2)-expressing zebrafish. Image specification: magnification $=5 x$, numerical aperture $=0.16$. (E) Representative western blot showing expression of human GM-CSF/CSF2 (hCSF2) and SCF/KITLG (hKITLG) in transgenic zebrafish. S1 and S2 denote samples from the transgenic larvae, and C1 and C2 are samples from control casper larvae. SCF/KITLG: stem cell factor/KIT ligand; GM-CSF/CSF2: granulocyte-monocyte colony- stimulating factor/colony-stimulating factor 2 . 
(dpi). We categorized each injected fish into one of the following categories: "no migration," "local dissemination," (dissemination within the yolk sac), and "migration" (distant migration beyond the yolk sac). There was no migratory difference between the cells injected into control versus CXCL12 fish (top panels, Figure 2A, B). However, expression of CXCL12 is low at this time point and is restricted to the posterior hemal arc near the tail. Previous studies have shown that DNA double-stranded breaks caused by either gamma irradiation or chemical agents, such as 5-fluorouracil or etoposide, can cause an increase in CXCL12 expression. ${ }^{33,34}$ Thus, we gamma-irradiated zebrafish larvae with a sublethal dose of radiation (15 Gy) 2 hours before transplantation and repeated the assay. We observed a drastic increase in the number of CXCL12 larvae that exhibited human T-ALL cell migration compared to the controls (bottom panels, Figure 2A, B). From the cells that migrated out of the yolk sac, we also saw hematopoietic niche-specific homing to the caudal hematopoietic tissue (CHT) at 144 hpf and later to the kidney marrow at $216 \mathrm{hpf}$ (Online Supplementary Figure S1).

For the transgenic fish expressing GM-CSF/CSF2 and SCF/KITLG (GS fish), we used CMK, a human Down syndrome acute myeloid leukemia (ML-DS) cell line for validation. While CMK cells survive in culture without additional cytokines, previous experiments in our hands demonstrated drastic cell death in zebrafish xenograft assays, suggesting that the cells are susceptible to their microenvironment. It was recently demonstrated that GM-CSF/CSF2 enhances survival in Down syndrome transient abnormal myelopoiesis (TAM), suggesting a growth advantage for ML-DS under GM-CSF/CSF2-rich conditions. ${ }^{35}$ When CMK cells were injected into GS larvae, xenografts demonstrated increased cell proliferation at 3 dpi compared to casper controls (Figure 2C, D). Strikingly, this was preceded by a sudden decrease in the number of CMK cells in both control and transgenic larvae at $2 \mathrm{dpi}$ (Figure 2D). These cells were injected into the yolk sac, an acellular environment, which may have resulted in delayed proliferation due to the restricted access of injected cells to circulating human cytokines. Thus, moving forward, all xenografts were performed by injection directly into the bloodstream of larval zebrafish, which is likely more relevant to adult human hematopoiesis. This strategy is in keeping with a recent emphasis on mechanochemical mechanisms, such as blood flow, leading to blood stem cell regulation. ${ }^{36}$

\section{Humanized zebrafish larvae show improved response to drug administration compared to controls}

Following our previous observation, we evaluated whether xenotransplantation of human cells into the circulation of GSS fish improved proliferation compared to that following yolk-sac injection. We injected human ML-DS CMK cells into both the yolk sac and the circulation of the larvae and observed a trend to increased proliferation of cells injected into the circulation compared to that of cells injected into the yolk sac (Online Supplementary Figure S2A). We then wanted to evaluate the fitness of our model in a preclinical drug-testing scenario. The ML-DS CMK cells were established from a 10-year old patient who responded to cytarabine. We wanted to determine whether this response was conserved in the context of GSS larvae. We injected CMK cells into the circulation of both GSS and casper larvae. The larvae were divided into two groups, and one group was treated with $1 \mathrm{mM}$ cytarabine 1 day-post injection. While there was no significant difference between the cytarabine administered and untreated casper groups $(P=0.94)$, the GSS larvae treated with cytarabine did show a significant decrease in the number of cells compared to the untreated control $(P=0.005)$ (Online Supplementary Figure S2B).

\section{GSS transgenic larvae show increased mortality compared to controls when transplanted with primary acute myeloid leukemia cells}

Following these validation experiments, both transgenic zebrafish lines were crossed to create a GSS (GM-

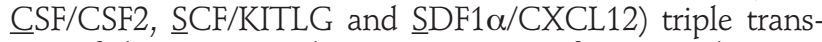
genic fish. We wanted to compare engraftment and expansion of primary patient-derived leukemias in the GSS larvae to controls. These xenografts were performed orthotopically by injecting primary AML cells into the circulation (common cardinal vein) at $72 \mathrm{hpf}$. We xenografted four distinct pediatric patient-derived AML samples: a $C B L$ exon 8 deletion with KMT2A-MLLT3 (MLL-AF9) fusion by karyotype (A23352); KRAS G12C point mutation (A23280), KMT2AMLLT3 fusion (AS12029811) and a ML-DS sample. Immediately post-injection, larvae were screened to select for a similar number of cells in both GSS and control groups of fish. We tracked the larvae until one of the groups reached $50 \%$ mortality and used the remaining larvae for targeted ECS) (Figure 3A). While the number of days required to reach $50 \%$ mortality varied across AML samples, the GSS fish consistently suffered greater mortality compared to control larvae (Figure 3A). This indicates increased cellular proliferation and leukemic burden, because when transplanted with human HSPC, both control and GSS larvae showed almost negligible death (Online Supplementary Figure S3). Since most leukemias are heterogeneous, they provide us with polymorphism or mutationspecific biomarkers that enable screening for clonal conservation. We prepared RNA from human AML xenografted control and GSS zebrafish and performed RNA-ECS to quantify clonal variability and conservation via the relative abundance of human leukemia-specific gene transcripts in the background of zebrafish transcripts. While some single nucleotide polymorphism variants were detected alternatively in the GSS or the control fish, overall the GSS fish retained a higher number of single nucleotide polymorphism variants representing more leukemic clones than the control fish. Mutations such as NOTCH (5094 C>T) and ALK (3375 C>A), which are silent and hence not pathogenic, were only present with a high allelic frequency $(>0.5)$ in GSS xenografts, suggesting the elimination of some clones in the controls (Figure 3B). Altogether, the data from both the control and GSS xenografts yielded 46 high confidence nucleotide variants, of which only $23(50 \%)$ were represented in controls compared to $42(93.4 \%)$ variants represented in the GSS zebrafish. This finding demonstrates that the GSS zebrafish provides a superior microenvironment for survival and expansion of human AML clonal diversity.

\section{The CXCR4-CXCL12 locus is dispensable for the migration of human leukemia cells to the caudal hematopoietic tissue but necessary for homing to the kidney marrow}

During validation of the CXCL12 transgenic zebrafish, we injected Jurkat cells into the yolk sac of $3 \mathrm{dpf}$ larvae, and a proportion of the cells transplanted into the transgenic larvae showed migration to the $\mathrm{CHT}$, a region equivalent to 
the fetal liver in humans. However, when we performed primary AML xenografts and injected these cells into the circulation, cells migrated to CHT uniformly in both control and transgenic larvae. Since the kidney marrow (KM), the zebrafish equivalent of human bone marrow, plays the role of the hematopoietic niche after migration from the CHT, we were curious to determine whether there was any difference in the migratory behavior to the KM between the GSS and casper larvae. We observed a profound difference in KM homing, with primary AML samples injected into the GSS larvae showing a propensity to migrate to the KM, which was not seen in controls (Figure 3C). In CXCL12 knock-out mouse models, hematopoietic stem cells migrated from the aorta-gonad-mesonephros to the fetal liver, but fetal liver to bone marrow homing was impaired. . $^{37,38}$ However, previous in vitro studies showed that hematopoietic stem cells from both fetal liver and bone marrow could respond to CXCL12 stimuli in a Boyden chamber assay. ${ }^{39}$ Together, these findings highlight that while the CXCR4CXCL12 axis is critical in bone marrow homing, it may be dispensable for fetal liver homing. We wanted to see if the homing of human leukemia cells to the zebrafish CHT was dependent on CXCL12-CXCR4. To do this, we returned to T-ALL, in which we had initially seen differential homing
A

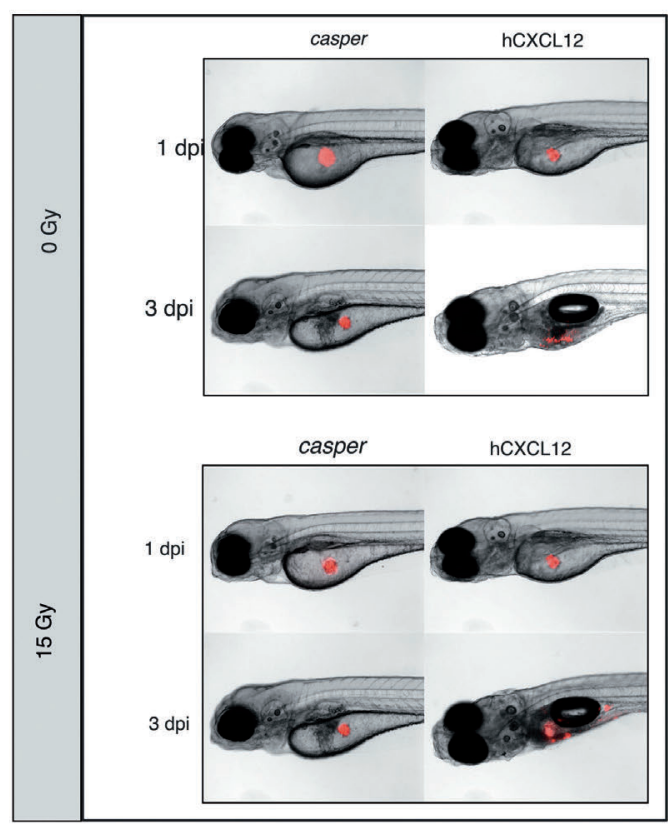

B

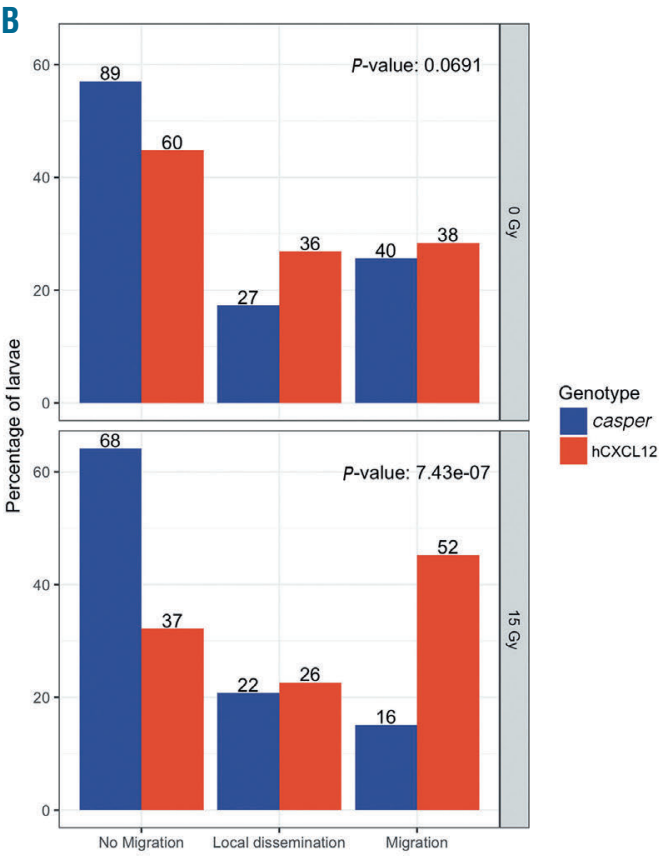

D
C

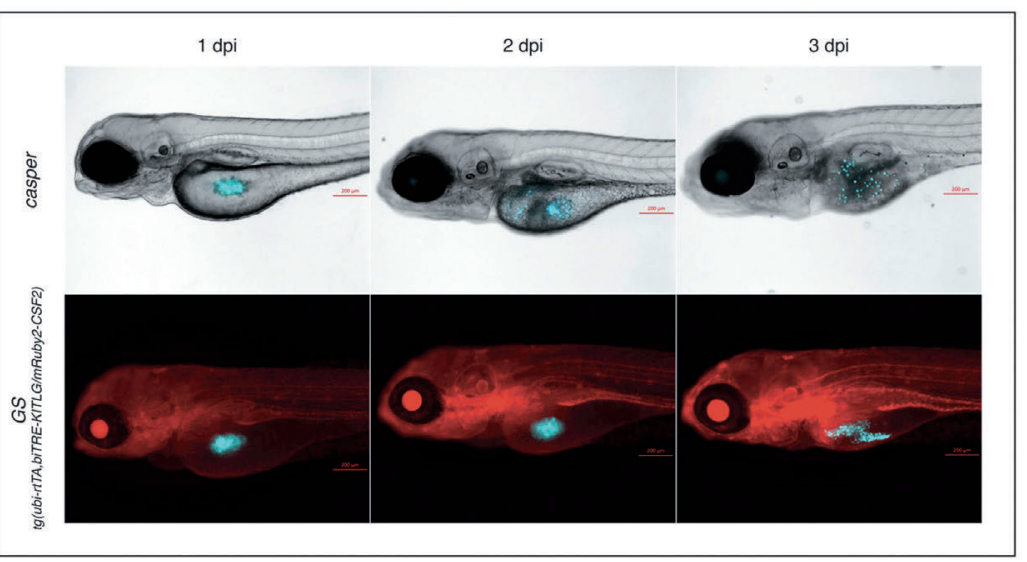

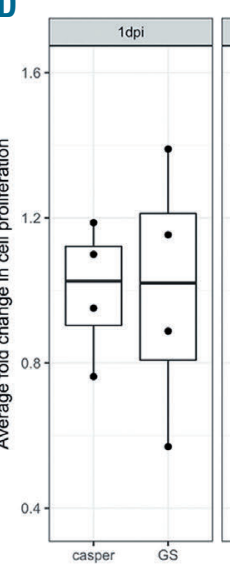
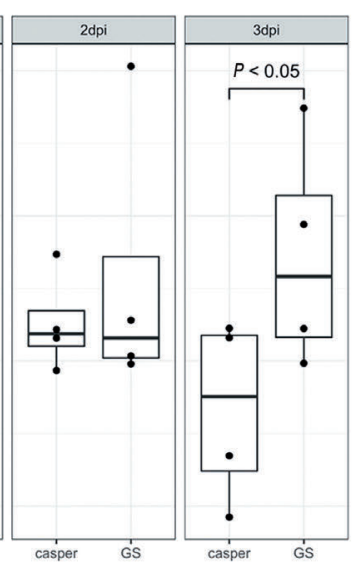

Figure 2. Irradiation of human CXCL12-expressing transgenic zebrafish dramatically increases cell migration, while myeloid leukemia cells exhibit enhanced proliferation in the presence of CSF2/GM-CSF and KITLG/SCF. (A) Human Jurkat cells (which highly express CXCR4, the receptor for CXCL12) were xenografted into control casper and CXCL12-expressing larvae, which were further divided into two groups: one which received 15 Gy irradiation and the other no irradiation at 72 hours postfertilization (hpf). Larvae were screened for cell migration at $144 \mathrm{hpf}$. Representative images of control and CXCL12-expressing larvae that were not irradiated uooer panel). Representative images of control and CXCL12-expressing larvae following 15 Gy gamma irradiation (lower panel). (B) Quantification of cell migration was classified into "no migration," "local dissemination" (dissemination within the yolk sac) and "migration" (distant migration beyond the yolk sac). Results represent three independent experiments. Numbers on the bar denote the total number of larvae per classification. (C) Representative images of zebrafish injected with CMK cells, a myeloid leukemia of Down syndrome (ML-DS) cell line. (D) Cell proliferation was quantified in transgenic larvae expressing GM-CSF/CSF2 and SCF/KITLG (GS fish) and casper controls following enzymatic digestion and dissociation at 1 day post-injection (dpi) (baseline), 2 dpi and 3 dpi. The analysis included fluorescence microscopy and cell counting. At $2 \mathrm{dpi}$ there was a slight decrease in cell numbers in both transgenic and control larvae. By $3 \mathrm{dpi}$ there was an increase in cell numbers in GS larvae, whereas cell numbers in control larvae decreased. Data presented represents four replicates with each dot in the bar graph denoting a single replicate. SCF/KITLG: stem cell factor/KIT ligand; GM-CSF/CSF2: granulocyte-monocyte colony-stimulating factor/colony-stimulating factor 2. 
using Jurkat cells, and this time employed a primary patient-derived T-ALL sample expressing very high levels of CXCR4. This sample was injected into the circulation of GSS and control larvae. The majority of the T-ALL cells in both control and GSS transgenic larvae migrated and stationed in the CHT, consistent with publications from other groups. ${ }^{40,41}$ However, in contrast to other zebrafish reports, ${ }^{40,41}$ following the addition of either a CXCR4-targeting antibody or isotype control, transplanted human T-ALL cells continued to migrate to the CHT (Online Supplementary Figure S4). These data are consistent with murine data and suggest that the CXCR4-CXCL12 axis does not contribute significantly to the migration of cells to the fetal liver.

Xenotransplanted of human hematopoietic stem cells and progenitor cells in GSS fish exhibit both enhanced self-renewal capacity and multilineage differentiation

Despite the widespread success of human tumor engraftment in zebrafish, normal tissue xenografts, including hematopoietic cells, have not been reported to engraft suc- cessfully. ${ }^{24}$ Given the enhanced proliferation observed for human leukemia samples transplanted into the GSS zebrafish, we wanted to determine if the presence of human cytokines could enhance human HSPC survival and differentiation in a zebrafish host. We collected umbilical cord blood from newly delivered infants and isolated lineage-depleted (lin) cells, which are highly enriched for human HSPC. These cells were fluorescently labeled with a cytoplasmic dye and transplanted into the circulation of both casper control and GSS larvae at $72 \mathrm{hpf}$. Consistent with previous reports, human HSPC did not survive past 24 hours in control larvae, ${ }^{24}$ but HSPC transplanted into GSS larvae continued to survive past 48 hours after which the cells began losing the cytoplasmic dye (Figure 4A). We extracted RNA for targeted exon sequencing from both control and GSS-injected larvae between 20-24 hpi. Of the three samples sequenced, only one sample injected into the casper larva had a detectable level of the human transcripts, in contrast to the GSS larvae, for which all three HSPC xenografted samples were found to have detectable human
A
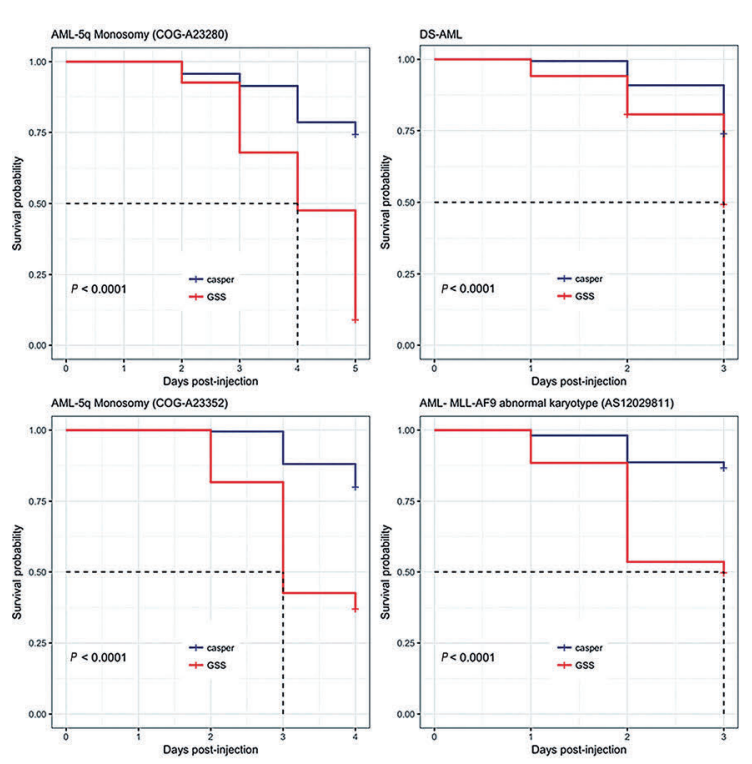

C
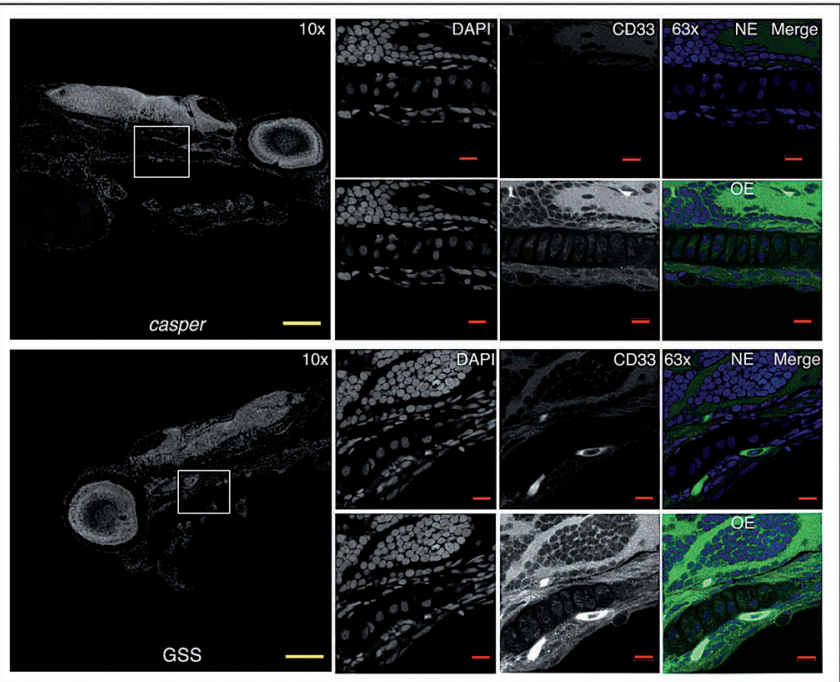

B

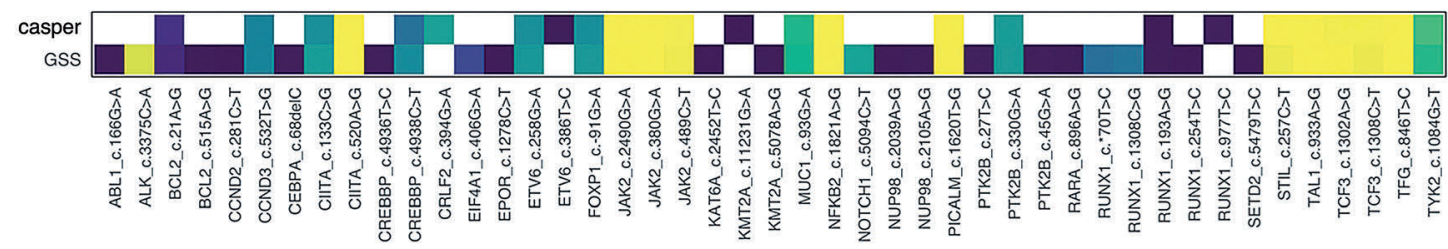

Allele Fraction

$\begin{array}{llll}0.25 & 0.50 & 0.75 & 1.00\end{array}$

Figure 3. Patient-derived acute myeloid leukemia transplantation into GSS transgenic larvae increases leukemia-related disease mortality and shows increased clonal representation in comparison to control larvae. (A) Kaplan-Meier curve showing increased acute myeloid leukemia (AML)-related mortality in GSS larvae transplanted with each of four different patient-derived AML samples compared with casper control larvae transplanted with the same samples $(P<0.0001)$. (B) Heatmap showing increased clonal representation in the GSS larvae compared to control larvae transplanted with the same sample as measured by RNA-error corrected sequencing. Different colors represent allele frequency from 0.002 (dark blue) to 1 (yellow). The white box represents an absence or allele frequency of less than 0.002. (C) Representative immunofluorescence images from sagittal zebrafish sections showing human CD33 ${ }^{+} \mathrm{AML}$ cells localized in the kidney marrow of the GSS transgenic fish. The left panel shows an overview of the fish section at 10x, and the white box highlights the region of interest. The top three right panels show images taken under normal exposure based on controls and the overexposed image shown in the middle of the lower three panels illustrates the kidney morphology. The parameters were kept constant between GSS and control sections during imaging $(\mathrm{N}=5)$. The yellow scale bar is equivalent to $100 \mu \mathrm{m}$, and the red scale bar is equivalent to $10 \mu \mathrm{m}$. The top panel shows images from kidney marrow of control larvae injected with human AML samples, where leukemia cells are not present, and the bottom shows GSS larvae with human $\mathrm{CD}_{3}{ }^{+} \mathrm{AML}$ cells. Image specifications: 10x images: numerical aperture $(\mathrm{NA})=0.45 ; 63 \mathrm{x}$ images: $\mathrm{NA}=1.4, \mathrm{Zoom}=$ 1.5. GM-CSF/CSF: granulocyte-monocyte colony-stimulating factor/colony-stimulating factor; SCF/KITLG: stem cell factor/KIT ligand; CXCL12/ SDF1 $\alpha$ :CXCL12/stromal cell derived factor $1 \alpha$; GSS: GM-CSF/CSF, SCF/KITLG, CXCL12/SDF1 $\alpha$-expressing transgenic zebrafish. 
transcripts present. To further determine if human transcripts were genuinely absent in the control fish, we performed another round of ECS-RNA library preparation and increased the starting total RNA from $100 \mathrm{ng}$ to 300-500 ng (depending on RNA availability). The absence of a detectable level of human transcripts was confirmed. Upon targeted transcriptome sequencing, we found that multilineage differentiation occurred in both GSS and control larvae, but with a myeloid bias. In the lymphoid lineage, only $B$ cells were sufficiently tractable, and transcripts of early $\mathrm{T}$ cell markers, CD3E and PTCRA, were absent, suggesting an absence of $\mathrm{T}$-cell differentiation (Figure $4 \mathrm{~B}$ ). In terms of selfrenewal capacity, HSPC transplanted into GSS larvae showed increased expression of both CD34 and GATA2 (Figure 4B). Specifically, there were 194 and 4,682 error-corrected transcripts of the human CD34 gene in $100 \mathrm{ng}$ total RNA input for control and GSS samples, respectively. While CD34 is a bona fide HSPC marker in hematopoietic cells,
GATA2 is required for the maintenance, generation, and survival of HSPC. ${ }^{42}$ The increased expression of CD34 and GATA2 suggests that HSPC undergo self-renewal only in the cytokine-rich context found in GSS larvae, but not in controls.

\section{Discussion}

While murine xenografts have provided essential insights into human leukemia pathogenesis, ${ }^{3,4,11}$ and despite even more excellent opportunities provided by ever more immunocompromised hosts, this model system continues to have limitations. Primary leukemia xenografts remain challenging in mice for many reasons, including intrinsic leukemic properties, absence or lack of bioactivity of some of the human factors found in the host microenvironment, and the presence of innate immune cells in the organism

A
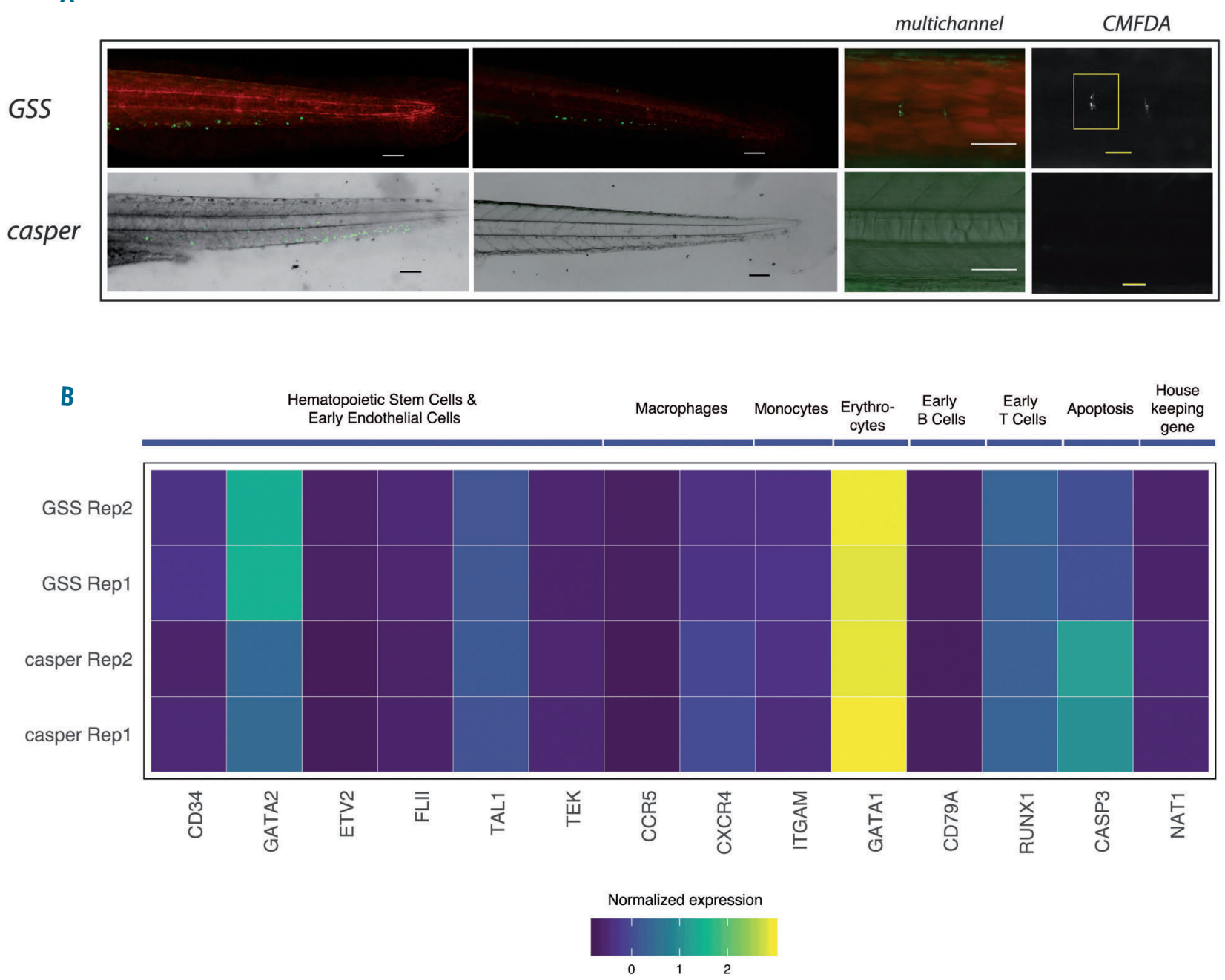

Figure 4. Umbilical cord blood-derived hematopoietic stem and progenitor cells show engraftment, self-renewal and multilineage differentiation in GSS larvae. (A) Representative image of GSS larvae and casper control larvae transplanted with umbilical cord blood (UCB)-derived hematopoietic stem and progenitor cells (HSPC). Image shows near complete absence of HSPC in control larvae at 28 hours post-injection (hpi), whereas the HSPC continue to survive in the GSS larvae at 28 hpi and HSPC are seen until $72 \mathrm{hpi}(\mathrm{N}=20$ /genotype). Image specification: magnification $=10 \mathrm{x}$, numerical aperture $=0.3$. White and black scale bars represent 100 $\mu \mathrm{m}$ and the yellow scale bar represents $50 \mu \mathrm{m}$. (B) Heatmap from RNA sequencing analysis of transplanted HSPC shows the increased expression level of self-renewal specific genes in HSPC transplanted into GSS larvae. Control and GSS larvae showed an identical expression of different lineage-specific genes. GM-CSF/CSF: granulocyte-monocyte colony-stimulating factor/colony-stimulating factor; SCF/KITLG: stem cell factor/KIT ligand; CXCL12/ SDF1 $\alpha$ :CXCL12/stromal cell derived factor $1 \alpha$; GSS: GM-CSF/CSF, SCF/KITLG, CXCL12/SDF1 $\alpha$-expressing transgenic zebrafish. 
that eliminate transplanted cells. Moreover, the complexity of these murine xenograft experiments, including the time to engraftment (typically 3-6 weeks), renders studies challenging to conduct in a high-throughput setting, and thus not readily amenable to clinically actionable drug screening experiments. Our group has pioneered human leukemia xenografts in zebrafish larvae and shown that this approach is amenable to medium-throughput drug screening in an actionable time-frame of $1-2$ weeks. ${ }^{17,18}$ However, the issue of conserved elements within the tumor microenvironment is also applicable, as illustrated by previous unsuccessful efforts to develop sustainable human HSPC zebrafish xenografts. ${ }^{24}$

To address the inherent limitations of the zebrafish microenvironment for sustaining human tumor xenografts, we created a humanized zebrafish model, the GSS fish, which expresses human CXCL12/SDF1 $\alpha$, SCF/KITLG, and GM-CSF/CSF2 to enhance human HSPC and patientderived leukemia engraftment to enable real-time preclinical therapeutic studies. As prior humanized mouse models informed our choice of cytokines, the GSS fish resembles the NSG-SGM3 mouse model, ${ }^{11,43}$ which expresses three cytokines with poor conservation between mouse and humans: GM-CSF, SCF, and IL-3. Zebrafish, being more evolutionarily distant, have only $20 \%$ conservation with the human CXCL12 ligand (Online Supplementary Figure $S 5 A, B)$ and considerable alteration in the CXCR4 binding region in humans (Online Supplementary Figure S5C). IL-3 is a critical factor in the expansion and chemotaxis of hematopoietic cells, but hematopoietic cytokines often perform redundant functions. For its expansion and homing function, IL-3 activates Raf/MEK/ERK signaling and small GTPases such as Rac and Ras. CXCL12 follows a very similar mechanism of action and additionally controls the fate of hematopoietic stem cells by restricting differentiation and enhancing stemness. ${ }^{44}$ GM-CSF, IL-3, and IL-5 also share a common $\beta$ chain that acts as a signaling subunit. ${ }^{45}$ GM-CSF also redundantly activates STAT and JAK2 pathways such as IL-3. ${ }^{46}$ So, we hypothesized that together SCF, GM-CSF, and CXCL12 would compensate for the absence of IL-3.

Previous observations revealed that even though there is minor to low cross-reactivity of human GM-CSF and SCF between mouse and humans, overexpression in the NSGS mice increased the number of mouse myeloid cells at the expense of erythropoiesis. ${ }^{43}$ Normally cytokine genes are expressed transiently and at low levels. Due to the use of powerful promoters and multiple integrations of the transgene, the quantity of cytokines secreted would be high in transgenic organisms and might lead to increased stress in the animals. Using a weak expression system will help overcome this phenomenon. The effect of prolonged exposure to human GM-CSF and SCF had not previously been studied in zebrafish, providing another reason for our choice of an inducible promoter. As observed when cells from the ML-DS line (CMK) were transplanted into GSS fish compared with casper controls, we observed enhanced proliferation supported by the cytokine milieu (likely predominantly GM-CSF, to which CMK cells are known to be responsive ${ }^{47}$ ) and a concomitant enhanced inhibition of proliferation following treatment with cytarabine.

Our results from primary AML cells regarding latency and mortality are consistent with findings from the NSGS mouse model, ${ }^{11}$ but the zebrafish model offers the benefit of medium- to high-throughput that can be applied in drug screening with survival as a readout. Furthermore, using ECS, we demonstrated that human AML transplanted into GSS larvae maintain better clonal representation than transplants into zebrafish lacking human cytokines. These findings are indicative of the GSS fish providing a more clinically representative microenvironment to that found in humans.

Given the results observed with patient-derived AML transplantation into GSS zebrafish, we hypothesized that these humanized zebrafish might provide an improved host environment for engrafting human blood stem cells, which to date has been a challenge in the zebrafish field. ${ }^{24}$ As previously demonstrated, umbilical cord blood-derived HSPC did not survive in control larvae but survived until 72 hpi in the GSS larvae, marking a substantive improvement over the current limit of 13 hours reported in the literature. ${ }^{25}$ ECS performed from these two different transplant populations revealed the upregulation of human genes specific to self-renewal only in the GSS larvae. The maintenance of this key stem cell characteristic exclusively in the GSS larvae affirms the utility of the "GSS human HSPC model" as a platform for studying drugs that enhance stem cell expansion in vivo. By contrast, we saw significant levels of all transcripts associated with multilineage differentiation, except for T-cell differentiation, in both control and GSS larvae. While the myeloid bias in GSS fish might be expected due to expression of GM-CSF and SCF, ${ }^{11,43}$ an absence of lymphoid cell differentiation in control larvae may be accounted for by the natural timeline of zebrafish lymphocyte development (the thymus does not appear until $5 \mathrm{dpf}$ ) and the lack of endogenous lymphoid-specific cytokines at this experimental time point. With growing interest in and efficacy of T-cell-mediated cancer immunotherapy and the absence of a fully functional adaptive immune system in zebrafish larvae until 1 month of age, this model is poised for further manipulation with respect to T-cell differentiation as a future platform for the preclinical testing of novel immunotherapy approaches using human cancer and HSPC co-transplantation.

In summary, through the generation of novel humanized zebrafish that express key hematopoietic cytokines, this study exploits the previously recognized imaging and higher-throughput screening advantages of the zebrafish model system to create a powerful new preclinical tool. The GSS fish, in conjunction with ECS bar-coding, can be used to screen for and also validate anti-leukemic and stem cell expanding therapeutics and contribute to the goal of providing biologically rational personalized treatment to patients.

\section{Acknowledgments}

The authors would like to thank David Malloy, Connor Booker, David Maley and Gretchen Wagner for zebrafish care and maintenance and Jennifer Curran for administrative support. This work was supported by a Canadian Cancer Society Research Innovation grant to JB, Kellsie's Hope Foundation and the Eli Seth Matthews Leukemia Foundation through a grant to TD, and a Discovery Grant from the Natural Sciences and Engineering Research Council of Canada (NSERC) to GD. GD and JB are Senior Scientists of the Beatrice Hunter Cancer Research Institute (BHCRI), and VR was funded by the Cancer Research Training Program of the BHCRI, with funds provided by the Terry Fox Research Institute through the Dr. Linnea Veinotte Memorial Graduate Student Award. VR was also funded by a Nova Scotia Health Research Foundation (NSHRF) Scotia Scholar award. 


\section{References}

1. Hidalgo M, Amant F, Biankin AV, et al. Patient-derived xenograft models: an emerging platform for translational cancer research. Cancer Discov. 2014;4(9):9981013.

2. Siolas D, Hannon GJ. Patient derived tumor xenografts: transforming clinical samples into mouse models. J Cancer Res. 2013;73(17):5315-5319.

3. Bonnet D, Dick JE. Human acute myeloid leukemia is organized as a hierarchy that originates from a primitive hematopoietic cell. Nat Med. 1997;3(3):730-737.

4. Lapidot T, Pflumio F, Doedens M, Murdoch B, Williams DE, Dick JE. Cytokine stimulation of multilineage hematopoiesis from immature human cells engrafted in SCID mice. Science. 1992;255 (5048):1137-1141.

5. Shlush LI, Zandi S, Mitchell A, et al. Identification of pre-leukaemic haematopoietic stem cells in acute leukaemia. Nature. 2014;506(7488):328-333

6. Larochelle A, Vormoor J, Hanenberg $\mathrm{H}$, et al. Identification of primitive human hematopoietic cells capable of repopulating NOD/SCID mouse bone marrow: implications for gene therapy. Nat Med. 1996;2(12):1329-1337.

7. Voskoglou-Nomikos T, Pater JL, Seymour L. Clinical predictive value of the in vitro cell line, human xenograft, and mouse allograft preclinical cancer models. Clin Cancer Res. 2003:9(11):4227-4239.

8. Brocker C, Thompson D, Matsumoto A, Nebert DW, Vasiliou V. Evolutionary divergence and functions of the human interleukin (IL) gene family. Hum Genomics. 2010;5(1):30-55.

9. Manz MG. Human-hemato-lymphoid-system mice: opportunities and challenges. Immunity. 2007;26(5):537-541.

10. Willinger $\mathrm{T}$, Rongvaux A, Takizawa $\mathrm{H}$, et al. Human IL-3/GM-CSF knock-in mice support human alveolar macrophage development and human immune responses in the lung. Proc Natl Acad Sci USA. 2011;108(6): 2390-2395.

11. Wunderlich M, Chou FS, Link KA, et al. AML xenograft efficiency is significantly improved in NOD/SCID-IL2RG mice constitutively expressing human SCF, GM-CSF and IL-3. Leukemia. 2010;24(10):1785-1788.

12. Morton JJ, Bird G, Keysar SB, et al. XactMice: humanizing mouse bone marrow enables microenvironment reconstitution in a patient-derived xenograft model of head and neck cancer. Oncogene. 2015;35(3):290300.

13. Rongvaux A, Willinger $\mathrm{T}$, Takizawa $\mathrm{H}$, et al. Human thrombopoietin knockin mice efficiently support human hematopoiesis in vivo. Proc Natl Acad Sci. 2011;108(6):23782383.

14. Traggiai E, Chicha L, Mazzucchelli L, et al. Development of a human adaptive immune system in cord blood cell-transplanted mice. Science. 2004;304(5667):104-107.

15. Coughlan AM, Harmon C, Whelan S, et al. Myeloid engraftment in humanized mice: impact of granulocyte-colony stimulating factor treatment and transgenic mouse strain. Stem Cells Dev. 2016:25(7):530-541.

16. Bryce PJ, Falahati R, Kenney LL, et al.
Humanized mouse model of mast cell mediated passive cutaneous anaphylaxis and passive systemic anaphylaxis. J Allergy Clin Immunol. 2016;138(3):769-779.

17. Bentley VL, Veinotte CJ, Corkery DP, et al Focused chemical genomics using zebrafish xenotransplantation as a preclinical therapeutic platform for T-cell acute lymphoblastic leukemia. Haematol. 2015.100(1):70-76

18. Corkery DP, Dellaire G, Berman JN. Leukaemia xenotransplantation in zebrafish-chemotherapy response assay in vivo. Br J Haematol. 2011;153(6):786-789.

19. Rajan V, Dellaire G, Berman JN. Modeling leukemogenesis in the zebrafish using genetic and xenograft models. Methods Mol Biol. 2016;1451:171-189.

20. Howe K, Clark MD, Torroja CF, et al. The zebrafish reference genome sequence and its relationship to the human genome. Nature. 2013;496(7446):498-503.

21. North TE, Goessling W, Walkley CR, et al. Prostaglandin E2 regulates vertebrate haematopoietic stem cell homeostasis. Nature. 2007;447(7147):1007-1011.

22. Liu Y, Asnani A, Zou L, et al. Visnagin protects against doxorubicin-induced cardiomyopathy through modulation of mitochondrial malate dehydrogenase. Sci Transl Med. 2014;6(266):266ra170

23. Stachura DL, Svoboda O, Campbell CA, et al. The zebrafish granulocyte colony stimulating factors (Gcsfs): two paralogous cytokines and their roles in hematopoietic development and maintenance. Blood. 2013;122(24):3918-3928.

24. Pruvot B, Jacquel A, Droin $\mathrm{N}$, et al. Leukemic cell xenograft in zebrafish embryo for investigating drug efficacy. Haematologica. 2011;96(4):612-616

25. Hamilton N, Sabroe I, Renshaw SA. A method for transplantation of human HSCs into zebrafish, to replace humanised murine transplantation models. F1000Res. 2018;7: 594

26. White RM, Sessa A, Burke C, et al. Transparent adult zebrafish as a tool for in vivo transplantation analysis. Cell Stem Cell. 2008;2(2):183-189.

27. Haldi M, Ton C, Seng WL, McGrath $P$ Human melanoma cells transplanted into zebrafish proliferate, migrate, produce melanin, form masses and stimulate angiogenesis in zebrafish. Angiogenesis. 2006;9(3):139-151.

28. Zhang $\mathrm{Y}$, Dépond $\mathrm{M}$, $\mathrm{He} \mathrm{L}$, et al CXCR4/CXCL12 axis counteracts hematopoietic stem cell exhaustion through selective protection against oxidative stress. Sci Rep. 2016;6:37827.

29. Greenbaum A, Hsu Y-MS, Day RB, et al. CXCL12 in early mesenchymal progenitors is required for haematopoietic stem-cell maintenance. Nature. 2013;495(7440):227230.

30. Sugiyama T, Kohara H, Noda M, Nagasawa T. Maintenance of the hematopoietic stem cell pool by CXCL12-CXCR4 chemokine signaling in bone marrow stromal cell niches. Immunity. 2006;25(6):977-988.

31. Peled A, Kollet O, Ponomaryov T, et al. The chemokine SDF-1 activates the integrins LFA-1, VLA-4, and VLA-5 on immature human CD34+ cells: role in transendothelial/stromal migration and engraftment of NOD/SCID mice. Blood. 2000;95(11):32893296.
32. Barretina J, Caponigro G, Stransky N, et al. The Cancer Cell Line Encyclopedia enables predictive modelling of anticancer drug sensitivity. Nature. 2012;483(7391):603.

33. Ponomaryov $\mathrm{T}$, Peled A, Petit I, et al Induction of the chemokine stromal-derived factor-1 following DNA damage improves human stem cell function. J Clin Invest. 2000;106(11):1331-1339

34. Glass TJ, Lund TC, Patrinostro X, et al. Stromal cell-derived factor-1 and hematopoietic cell homing in an adult zebrafish model of hematopoietic cell transplantation. Blood. 2011;118(3):766-774.

35. Labuhn M, Perkins K, Papaemmanuil E, et al. Modelling the progression of a preleukemic stage to overt leukemia in children with Down syndrome. Blood. 2018; 132(Suppl 1):543.

36. Theodore LN, Lundin V, Wrighton PI, et al YAP regulates hematopoietic stem cell formation in response to the biophysical forces of blood flow. Blood 2017;130(Suppl 1): 1147

37. Zou Y-R, Kottmann AH, Kuroda M, Taniuchi I, Littman DR. Function of the chemokine receptor CXCR4 in haematopoiesis and in cerebellar development. Nature. 1998;393(6585):595-599.

38. Nagasawa T, Hirota S, Tachibana K, et al. Defects of B-cell lymphopoiesis and bonemarrow myelopoiesis in mice lacking the CXC chemokine PBSF/SDF-1. Nature. 1996;382(6592):635-638

39. Christensen JL, Wright DE, Wagers AJ, Weissman IL. Circulation and chemotaxis of fetal hematopoietic stem cells. PLOS Biol. 2004;2(3):e75.

40. Tulotta C, Stefanescu C, Beletkaia E, et al. Inhibition of signaling between human CXCR4 and zebrafish ligands by the small molecule IT1t impairs the formation of triple-negative breast cancer early metastases in a zebrafish xenograft model. Dis Model Mech. 2016;9(2):141-153.

41. Sacco A, Roccaro AM, Ma D, et al. Cancer cell dissemination and homing to the bone marrow in a zebrafish model. Can Res. 2016;76(2):463-471.

42. de Pater E, Kaimakis P, Vink CS, et al. Gata2 is required for HSC generation and survival. J Ex Med. 2013;210(13):2843-2850

43. Nicolini FE, Cashman JD, Hogge DE, Humphries RK, Eaves CJ. NOD/SCID mice engineered to express human IL-3, GM-CSF and Steel factor constitutively mobilize engrafted human progenitors and compromise human stem cell regeneration. Leukemia. 2003;18(2):341-347.

44. Arai A, Jin A, Yan W, et al. SDF-1 synergistically enhances IL-3-induced activation of the Raf-1/MEK/Erk signaling pathway through activation of Rac and its effector Pak kinases to promote hematopoiesis and chemotaxis. Cell Signal. 2005;17(4):497506.

45. Rossjohn J, McKinstry WJ, Woodcock JM, et al. Structure of the activation domain of the GM-CSF/IL-3/IL-5 receptor common $\beta$ chain bound to an antagonist. Blood. 2000;95(8): 2491-2498.

46. Ihle JN. Cytokine receptor signalling. Nature. 1995;377(6550):591-594

47. Komatsu N, Suda T, Moroi M, et al. Growth and differentiation of a human megakaryoblastic cell line, CMK. Blood. 1989;74(1): $42-48$ 